\title{
Phronesis
}

\section{Le rôle du chercheur dans l'accompagnement des enseignants en pédagogie Freinet : questionnement autour de l'entretien d'auto-confrontation}

\section{The researcher's role in accompanying teachers in Freinet pedagogy: questioning about self-confrontation interview}

\section{Catherine BOYER et Maria PAGONI}

Volume 6, numéro 1-2, 2017

Les recherches collaboratives en éducation et en formation : référents théoriques, outils méthodologiques et impacts sur les pratiques professionnelles

URI : https://id.erudit.org/iderudit/1040222ar

DOI : https://doi.org/10.7202/1040222ar

Aller au sommaire du numéro

Éditeur(s)

Université de Sherbrooke

Champ social éditions

ISSN

1925-4873 (numérique)

Découvrir la revue

Citer cet article

BOYER, C. \& PAGONI, M. (2017). Le rôle du chercheur dans l'accompagnement des enseignants en pédagogie Freinet : questionnement autour de l'entretien d'auto-confrontation. Phronesis, 6(1-2), 110-125.

https://doi.org/10.7202/1040222ar
Résumé de l'article

Les auteures interrogent dans cet article un moment précis d'une recherche collaborative qui vise à analyser les pratiques de trois enseignants d'une école primaire se réclamant de la pédagogie Freinet. Ces pratiques concernent le dispositif de recherche documentaire (RD) expérimenté pour la première fois par les enseignants. L'analyse est centrée sur le moment d'entretien

d'auto-confrontation collectif et propose deux axes de réflexion : une réflexion sur le questionnement des chercheurs et leurs effets sur la construction des savoirs disciplinaires des enseignants ; une réflexion sur les controverses qui font leur apparition pendant le moment de l'entretien. Les auteures montrent dans cet article que ces controverses constituent à la fois l'objet de recherche de ce collectif et l'enjeu professionnel de ce dispositif innovant. 


\title{
Le rôle du chercheur dans l'accompagnement des enseignants en pédagogie Freinet : questionnement autour de l'entretien d'auto-confrontation.
}

\author{
Catherine BOYER et Maria PAGONI
}

Université Lille 3

Laboratoire CIREL, équipe Théodile-CIREL

Domaine Universitaire Pont de Bois

BP 60149, 59653 Villeneuve d'Ascq Cedex

catherine.boyer@univ-lille3.fr

maria.pagoni-andreani@univ-lille3.fr

Mots-clés : recherche documentaire, pédagogie Freinet, recherche collaborative, entretien d'auto-confrontation.

Résumé : Les auteures interrogent dans cet article un moment précis d'une recherche collaborative qui vise à analyser les pratiques de trois enseignants d'une école primaire se réclamant de la pédagogie Freinet. Ces pratiques concernent le dispositif de recherche documentaire (RD) expérimenté pour la première fois par les enseignants. L'analyse est centrée sur le moment d'entretien d'auto-confrontation collectif et propose deux axes de réflexion : une réflexion sur le questionnement des chercheurs et leurs effets sur la construction des savoirs disciplinaires des enseignants; une réflexion sur les controverses qui font leur apparition pendant le moment de l'entretien. Les auteures montrent dans cet article que ces controverses constituent à la fois l'objet de recherche de ce collectif et l'enjeu professionnel de ce dispositif innovant.

\section{Title : The researcher's role in accompanying teachers in Freinet pedagogy: questioning about self- confrontation interview.}

Keyword : documentary research, Freinet pedagogy, collaborative research, self-confrontation interview.

Abstract : The article examines a specific time of a collaborative research which analyzes the practices of three teachers of a primary school claiming to Freinet pedagogy. These practices concern students coaching in documentary research for the first time experienced by teachers. The analysis focuses on the moment of self-confrontation interview and offers two lines of reflection: a reflection on the researchers questions and their effects on the construction of subject knowledge of teachers; a reflection on the controversies that appear during the time of the interview. The article shows that these controversies constitute both the research object of this collaborative group and the professional challenge of this innovative device. 


\section{Dhéronestece}

\section{Introduction}

Nous proposons d'analyser dans cet article la dernière phase d'un dispositif de collaboration entre chercheurs et professionnels mis en place pour analyser les pratiques de trois enseignants d'une école primaire se réclamant de la pédagogie Freinet (PF). Les pratiques étudiées présentent la spécificité de constituer une expérimentation pédagogique pour les enseignants eux-mêmes qui expérimentent un dispositif de recherche documentaire, complètement nouveau pour eux, composé de trois types de tâches, pour les élèves : question de départ - recherche de documents - exposé en classe devant les élèves. La question posée est la suivante: Comment un entretien d'auto confrontation collectif contribue-t-il à susciter une analyse réflexive des situations professionnelles et donc le développement professionnel ? Quel est le rôle du chercheur dans ce dispositif et comment les professionnels se l'approprient-ils?

II s'agit là d'une nouvelle question de recherche nécessitant la relecture de notre corpus, cela n'étant pas un des questionnements à l'origine de la recherche collaborative.

Pour répondre à ces questions, nous définissons d'abord la notion du développement professionnel et présentons le cadre théorique dans lequel il s'inscrit. Nous décrivons ensuite le dispositif de recherche collaborative et les critères d'analyse de l'entretien d'auto confrontation selon les perspectives de la clinique de l'activité, pour terminer par l'analyse de la posture des chercheurs et des effets obtenus pendant ce moment de l'entretien.

\section{Deux termes clés : Développement professionnel et recherche collaborative}

\subsection{Quelle définition du développement professionnel ?}

Notre recherche s'inscrit dans une préoccupation générale concernant les conditions du développement professionnel des enseignants s'inscrivant dans une pédagogie alternative telle que la pédagogie Freinet. Ce développement professionnel renvoie pour nous à l'effet de formation visé dans le cadre d'une recherche collaborative, d'où la pertinence de préciser sa signification. Il est ici abordé à partir d'une théorie de l'activité empruntant des éléments à la théorie des champs conceptuels (Vergnaud 1990) et à la didactique professionnelle (Pastré 2011). Selon cette définition, l'activité est organisée par des schèmes d'action qui révèlent des constantes dans la conduite du sujet chaque fois qu'il se trouve devant une classe de situations (des situations qui présentent des caractéristiques semblables). Ces schèmes sont susceptibles d'être reproduits d'une situation à l'autre tout en s'adaptant aux particularités de chacune d'elles. Les schèmes d'action se structurent par des invariants opératoires, leur noyau central, conçus comme des unités implicites (en-acte) ou explicites sur lesquelles s'appuie le sujet pour construire la signification d'une situation et agir. Ces invariants peuvent être des représentations et convictions, des principes d'action (théorèmes en acte et concepts en acte) ou encore des composantes affectives liées à l'identité professionnelle des acteurs (Vinatier 2009).

Cette définition générale de l'activité ouvre trois pistes de réflexion sur le développement professionnel qui vont nous préoccuper dans le présent article :

- La première concerne la distinction entre les invariants opératoires qui sont spécifiques à chaque acteur et marquent sa singularité et ceux qui acquièrent le statut de normes ancrées dans l'histoire du métier et la culture du groupe d'appartenance. Cette distinction n'est pas statique mais dynamique et révèle les nœuds de débat et de négociation de la signification des situations par des acteurs, qui constituent autant de controverses caractérisant le métier selon le vocabulaire utilisé par la clinique de l'activité (Clot, 1999).

- La deuxième réflexion concerne la place des médiations symboliques dans les déplacements observés auprès des invariants opératoires des acteurs : prise de conscience, formalisation, transformation de ces invariants d'outils en 
objet de l'activité. Plusieurs dispositifs d'analyse réflexive des pratiques par la médiation des pairs ou des entretiens spécifiques ont été développés ces dernières années dans la lignée des travaux de Schön (1983) ou de Vygotsky (1985), selon les courants et les postures de recherche adoptées. Ces travaux renforcent les liens entre recherche et formation et reposent sur l'idée que le dispositif de recherche basé sur l'analyse réflexive des acteurs peut avoir des effets sur le développement professionnel de ces derniers.

- La troisième réflexion concerne la nature des savoirs professionnels qui vont se construire à partir de l'analyse de l'activité des professionnels. Nous partons du postulat que ces savoirs ont une double nature : nature expérientielle, liée à la formalisation des invariants opératoires qui organisent l'activité des professionnels, mentionnés plus haut; nature épistémique liée aux modèles théoriques de l'activité d'enseignement et d'apprentissage qui alimentent la recherche. Ces deux aspects des savoirs se négocient pendant la recherche collaborative et déterminent le rôle de chacun des participants dans son déroulement.

\subsection{Quelle recherche collaborative?}

Les collaborations entre chercheurs et professionnels ont connu une croissance accrue ces dernières années dans le secteur des sciences de l'éducation et de la formation aussi bien en France qu'au Québec (Kahn, Hersant, Orange-Ravachol 2010, Morrissette 2013, Morrissette \& Desgagné 2009). Ce phénomène révèle un besoin de rapprochement entre recherche - formation - travail expliqué par des raisons à la fois politico-économiques (améliorer la relation formation - emploi, questionner le caractère opératoire de la recherche), pédagogiques (former des praticiens réflexifs susceptibles de mettre en place un processus de développement professionnel continu) et scientifiques / épistémologiques (saisir la réalité du travail des acteurs, questionner les rapports entre savoirs académiques et savoirs professionnels, aborder l'expérience comme source d'apprentissage ...).

Cette forme de collaboration n'est pas nouvelle puisque la tradition de la recherche - action incitant les professionnels à participer à la mise en place et à l'évaluation des pratiques " ordinaires » et / ou expérimentales puise ses racines dans les années 1940 et plus précisément dans les travaux de Lewin, psychologue expérimental allemand. Ce dernier a montré que la recherche-action pouvait en même temps aboutir à des savoirs théoriques et à des changements sociaux (Catroux 2002). En faisant la distinction entre recherche-action et recherche collaborative, Morrissette (2013) rappelle comment la référence aux travaux de Schön (1983) a conduit à une autre forme de collaboration entre chercheurs et professionnels basée sur l'activité réflexive de ces derniers.

Nous utilisons ici comme point de départ la définition de la recherche collaborative proposée par Desgagné (1997) selon trois caractéristiques :

- Co-construction d'un objet de connaissance entre un chercheur et des praticiens. Cela signifie « que les praticiens s'engagent, avec le chercheur, à explorer un aspect de leur pratique et que l'objet même de la recherche porte sur leur compréhension en contexte du phénomène exploré. L'objet de la recherche collaborative s'appuie sur une conception du professionnel comme d'un « acteur social compétent ", c'est-à-dire un acteur qui exerce un « contrôle réflexif » sur son contexte de pratique » (Desgagné 1997, p.383).

- Visée à la fois de production de connaissances et de développement professionnel : "L'activité d'exploration se présente sous deux facettes: pour le chercheur qui en fait un objet d'investigation, elle est activité de recherche et pour les praticiens qui en font une occasion de perfectionnement, elle est activité de formation. Le chercheur susceptible d'encadrer l'activité doit par conséquent cumuler les rôles de chercheur et de formateur » (lbid., p.384).

- Visée d'une médiation entre communauté de recherche et communauté de pratique. Cette médiation peut s'opérer par le rapprochement des concepts utilisés dans les deux communautés, les cadres de réflexion et de questionnement, les priorités et les valeurs.

Nous avons choisi de mettre en place un protocole de recherche collaborative inspiré de la clinique de l'activité. La particularité de cette approche est qu'elle s'appuie sur l'analyse réflexive de l'activité d'un collectif de professionnels à travers un dispositif d'entretien, l'auto-confrontation (simple, croisé et collectif) qui s'organise selon les étapes suivantes:

- La constitution d'un groupe d'analyse et du matériau : pendant cette phase se constitue le groupe de travail comportant à la fois des professionnels et des chercheurs (Faïta, 2001, cité par Faïta \& Vieira, 2003). Les deux parties se mettent d'accord sur l'objet du travail et sur leur volonté commune de travailler ensemble. 
- La réalisation des auto-confrontations : pendant cette phase le(s) participant(s) se confronte(nt) aux images filmées et les commente(nt). L'auto-confrontation est d'abord simple (c'est le professionnel filmé qui commente sa propre activité), puis croisée (les séquences sont commentées par le professionnel lui-même et un autre de ses collègues). On peut aussi mettre en place des auto-confrontations collectives où les séquences filmées sont commentées par un groupe de professionnels.

- L'extension de l'analyse à l'ensemble du collectif professionnel : cette phase concerne l'analyse des séquences et entretiens enregistrés. Cette analyse peut se faire en collaboration avec les professionnels ou, au cas échéant, elle peut être soumise aux professionnels pour avoir leur avis.

Nous retrouvons dans ces étapes le rôle médiateur de la recherche dans le développement professionnel des acteurs, cité plus haut dans le modèle de Desgagné. Cette médiation est formulée par Yves Clot et ses collaborateurs, de la façon suivante : " nous choisissons de faire, avec les collectifs de travailleurs, une co-analyse qui mise sur un développement aussi bien des sujets, du collectif que de la situation ». À partir d'enquêtes menées dans différents secteurs professionnels, les auteurs aboutissent au constat suivant : " seuls ces collectifs eux-mêmes peuvent opérer des transformations durables de leur milieu de travail. » Dans cette optique, notre tâche est de « seconder leurs efforts pour élargir leur propre rayon d'action, c'est-à-dire le développement de leur activité qui est l'objet même de notre recherche. » (2000, p.1).

Notre réflexion porte plus précisément sur ce rôle du chercheur, qui propose un dispositif de réflexion permettant aux praticiens d'analyser leurs propres pratiques et de les faire évoluer. Quelle est la posture du chercheur dans le dispositif de l'autoconfrontation ? En quoi consiste exactement son rôle de médiateur et comment est-il géré pendant l'entretien ? En quoi consiste le « bricolage » du chercheur, c'est-à-dire sa capacité d'adaptation à la dynamique de la situation d'interaction ?

\section{La méthodologie de recherche : les dilemmes et les choix des chercheurs}

D'abord, nous situons la recherche collaborative dans son contexte spatio-temporel, puis présentons le dispositif de recherche documentaire (RD) sur lequel portent les pratiques observées, développons la construction du questionnement de la recherche et la méthode d'entretien d'auto-confrontation collectif.

\subsection{Le contexte de la recherche collaborative : Le dispositif de la Recherche Documentaire (RD)}

Le point de départ de cette recherche renvoie à une préoccupation à la fois politique et pédagogique des enseignants de la pédagogie Freinet. Organisés autour de l'ICEM (Institut Coopératif de l'École Moderne), ces enseignants sont très impliqués dans le développement de leurs pratiques et ont eux-mêmes constitué un laboratoire de recherche basé sur des fréquentes rencontres d'analyse des pratiques. La collaboration avec la recherche est récente et a progressivement évolué. Les chercheurs ont initialement eu une fonction d'évaluation des résultats obtenus par cette pédagogie (Reuter, 2007). Ils ont ensuite participé à l'analyse de certaines pratiques d'enseignement (comme les pratiques dans les conseils de coopérative, Pagoni 2014). Ils ont finalement été sollicités pour accompagner les enseignants dans l'élaboration de nouveaux dispositifs. Ainsi, une relation d'enrichissement mutuel s'est construite progressivement entre la communauté des chercheurs et la communauté de ces professionnels.

La recherche se déroule pendant l'année universitaire 2010 - 2011 dans une école primaire de la région parisienne se réclamant de la pédagogie Freinet (PF). Trois enseignants ( $\mathrm{Sab}, \mathrm{Am}$ et $\mathrm{Em}$ ) ont expérimenté pendant un an un dispositif de RD qu'ils ont construit ensemble. Ces enseignants ont des classes mixtes du cycle 3 ( 9 - 11 ans). Le dispositif suit l'organisation suivante :

- L'émergence du questionnement des élèves se fait dans la classe. Les élèves proposent des questions qui vont constituer par la suite les objets de leur RD. Ces questions peuvent émerger d'autres moments d'enseignement ou encore pendant les moments d'exposé des élèves. Les élèves choisissent un thème qui se déroule en plusieurs questions auxquelles ils doivent trouver des réponses. Ils écrivent leurs questions sur une feuille de papier. L'originalité de ce dispositif par rapport à l'enseignement traditionnel est qu'il y a un temps spécifique consacré au questionnement transversal des élèves sans qu'il soit attribué à telle ou telle discipline d'enseignement. L'institution d'un moment de $\mathrm{RD}$ en soi répond à un des objectifs centraux de la PF qui est de développer auprès de l'élève l'observation critique de son environnement. 
- La recherche documentaire constitue le moment où les élèves font des recherches pour répondre à leurs questions. Ces recherches peuvent se faire dans les livres et encyclopédies au centre de documentation de l'école et éventuellement dans la salle informatique. L'enseignant qui accompagne les élèves n'est pas celui de la classe de l'élève mais un enseignant, parmi les trois, qui est chargé de la RD et qui change toutes les quatre semaines. La difficulté de ce système consiste au fait que l'enseignant chargé de la RD ne connaît pas le contexte de l'émergence du questionnement de l'élève et qu'il est obligé de s'en construire une représentation. Pendant cette phase qui peut durer quelques semaines, l'élève doit faire le choix des documents dont il a besoin, les lire, sélectionner les informations, puis les écrire de façon synthétique sur une feuille en illustrant éventuellement sa présentation par une ou deux images.

- La présentation de l'exposé se fait à nouveau dans la classe de l'élève, en situation collective. Une fois l'exposé effectué, la classe pose des questions, ce qui peut constituer le point de départ d'un nouvel exposé.

\subsection{La construction de l'objet de recherche et du groupe d'analyse}

Le point de départ de la recherche était le besoin des enseignants de se faire accompagner dans l'analyse du dispositif présenté ci-dessus avec l'objectif de le rendre plus systématique et efficace. Ce besoin a suscité l'intérêt des trois chercheurs M, $\mathrm{C}$ et $\mathrm{R}$ qui se situent au croisement de deux approches :

- Approche de l'analyse du travail des enseignants (M et $\mathrm{C}$ ) de la PF dans la continuité des travaux de recherche déjà effectués par l'équipe Théodile-CIREL (Reuter 2007).

- Approche didactique des modalités d'enseignement et d'apprentissage orientée vers deux aspects intéressants de cette expérimentation : le rôle de ce dispositif dans le croisement de plusieurs disciplines scolaires ( $M, C$ et R); les liens plus spécifiques avec la didactique des sciences à l'école ainsi que de l'oral particulièrement concerné par la phase de l'exposé (C et R).

On peut faire l'hypothèse que ces ancrages théoriques ont joué un rôle dans le questionnement des enseignants-chercheurs comme nous allons le voir plus loin ainsi que dans la construction de l'objet de recherche tel qu'il s'est négocié avec les enseignants. Ils sont pourtant restés implicites dans les interactions avec les enseignants et n'ont pas donné lieu à des séances de formation particulières.

\subsection{Le recueil des données}

Pour analyser l'activité des enseignants (objet initial de la recherche collaborative), nous nous sommes appuyées sur plusieurs types de données parmi lesquels nous en retenons deux pour la présente analyse:

- Des observations en classe pour documenter les pratiques effectives des enseignants et les utiliser comme support d'analyse de leur activité. Ces observations ont été faites en binôme de chercheurs et filmées avec recueil des productions des élèves et supports utilisés. Nous souhaitions disposer pour chacun des 3 enseignants de vidéo des trois phases de la RD (questionnement ; recherche dans les documents ; exposé) et observer l'intégralité de la RD pour quelques-uns des élèves de ces 3 classes.

- Un entretien d'auto-confrontation collectif effectué à partir des extraits de séances présentés à la fin de l'année scolaire, séances menées par les trois enseignants à différents moments de la RD (questionnement; recherche dans les documents ; exposé).

Cet article étant centré sur l'analyse de l'entretien d'auto-confrontation collectif, nous allons focaliser notre attention sur la mise en place de ce moment du dispositif. Précisons à ce propos que les chercheurs ont pris des garanties (Faïta \& Vieira 2003) concernant le choix des moments à filmer. En accord avec les professionnels, nous avons filmé plusieurs moments de RD, qui se situent au croisement des critères suivants :

- Correspondre aux trois phases du dispositif (questionnement, recherche, exposé) ;

- Permettre d'avoir des traces de l'activité de chacun des enseignants pendant les trois phases ; 
- Permettre d'avoir le parcours de quelques élèves du début jusqu'à la fin de leur recherche (avec des enseignants différents).

\subsection{Un moment particulier : I'entretien d'auto-confrontation collectif}

L'entretien mis en place est un entretien d'auto-confrontation collectif qui présente certaines spécificités par rapport à ce qui est envisagé par la clinique de l'activité :

- Le nombre des professionnels de terrain : il se fait avec trois enseignants qui participent à ce dispositif, auxquels se rajoute la présence de la directrice de l'école qui avait inspiré ce groupe de travail et permis mise en place de cette recherche collaborative.

- Le nombre des enseignants-chercheurs que nous nommerons pour simplifier « chercheurs » : il y en a trois, ce qui peut entraîner, malgré la mise en place d'un protocole commun d'intervention, une disparité dans les modalités et le contenu des interventions.

- Les liens avec les autres phases de la recherche collaborative. Selon les principes de la clinique de l'activité, l'autoconfrontation croisée se situe entre l'auto-confrontation simple qui se fait en individuel avec le chercheur et le retour de ce travail à l'ensemble du collectif. Or, l'entretien que nous avons mis en place combine ces trois phases en une seule par manque de temps des professionnels. Ainsi, malgré le fait que nous donnons la consigne que "le professionnel concerné commente d'abord la séance filmée, puis intervient l'ensemble du groupe », les enseignants risquent de se trouver dans une position de justification qui transforme les invariants opératoires investis dans l'action : "On doit rester vigilant face au danger d'instrumentalisation de ce premier support, repris et développé pendant l'autoconfrontation croisée, car le risque existe que le vouloir dire des protagonistes se substitue déjà à leur activité » (Faita \& Vieira 2003, p.130).

- Le choix des extraits vidéo. Il est fait par les chercheurs et repose sur deux visées de cette recherche collaborative. Une première visée est l'explicitation de moments clé qui sont importants dans la RD (l'émergence de questionnement, la recherche documentaire, l'exposé). Ces extraits sont choisis de manière à susciter une posture réflexive chez les enseignants pour les inciter à verbaliser les choix plus ou moins conscients posés durant ces moments (théorèmes et concepts en acte). Une seconde visée est de faire émerger les zones d'ombre de leur pratique qui semblent ne pas être pris en compte dans leur accompagnement et qui seraient susceptibles de faire obstacle pour les élèves, selon les hypothèses et les ancrages théoriques des enseignants-chercheurs. Par exemple, le choix de l'extrait concernant la recherche dans un livre repose sur l'hypothèse du chercheur que la difficulté de conceptualisation de l'index ou de la table des matières, par les élèves, n'est pas perçue par les enseignants. Il y aurait, en d'autres termes, une supposée transparence du rôle des images dans le lecture du livre, qui serait illusoire et ferait obstacle aussi bien à la lecture du document qu'à l'écriture des exposés : complexité du lexique qui s'appuie sur des aspects conceptuels très développés (par exemple pour les régimes alimentaires des dinosaures "vite à table » et "les autres herbivores »), mais aussi complexité des relations entre les différents messages iconiques (images, dessins, schémas, photos..) et les textes à lire. Nous retrouvons ici le rôle du chercheur dans l'articulation des aspects de recherche et de formation des recherches collaboratives : "Il incombe donc au chercheur, partiellement, parfois totalement, d'envisager le choix de moments et séquences de l'activité en fonction de cette activité - plus exactement de la perception qu'il en a - plus que de la représentation discursive que le collectif est disposé à en fournir » (Faïta \& Vieira 2003, p.127).

\section{Les axes d'analyse de l'entretien d'auto-confrontation collectif}

Notre objectif est d'analyser le moment d'auto-confrontation collectif comme un moment d'interaction verbale et porteur de développement professionnel selon trois axes d'analyse :

- Distinction des thèmes abordés durant les échanges (découpage thématique) et de leur imbrication sous forme d'un synopsis (cf. annexe). Le synopsis consiste à reconstituer les situations professionnelles à partir de l'observation des séances de classe et les extraits vidéo choisis par le chercheur. A partir du découpage thématique et chronologique, 
nous reconstruisons les questions qu'ils se posent sur leurs pratiques professionnelles.

- Les rôles et les places de chacun des participants dans les échanges. Le rôle de questionnement du chercheur (types de questions, reformulations, commentaires éventuels) et les effets observés exemplifiés.

- Les points communs et les controverses observés dans la formulation des savoirs professionnels nécessaires à l'accompagnement des élèves dans cette activité.

\subsection{Découpage des épisodes}

Nous avons découpé l'entretien en nous appuyant sur la méthode du synopsis (Schneuwly \& al 2006) qui distingue des unités d'analyse, les épisodes, selon les activités en jeu et les interactions en cours (cf. annexe). Dans cette recherche, nous estimons que les extraits vidéo présentés permettent l'avancée des échanges et de fait c'est l'objet de l'enseignement qui devient le support des interactions.

Nous présentons par la suite la structure générale de l'entretien en regroupant les 32 épisodes identifiés en cinq grandes parties (représentants les extraits visionnés) ainsi que l'introduction de la démarche et de la clôture de l'entretien. Ces parties révèlent en même temps les points forts de cette activité qui constituent autant des points de départ des controverses qui vont apparaître entre les enseignants et qui suscitent le cœur de cette activité, comme nous allons le voir plus loin :

\section{L'émergence du questionnement de l'élève en RD (9 épisodes de 4 à 139)}

Ces neuf épisodes développent le point de vue d'Am qui présente sa gestion et sa prospection sur ce moment, suivi du point de vue de Sab et Em où elles abordent leur gestion individuelle. Une réflexion sur l'accompagnement à la construction de protocoles clôt cette partie.

\section{L'accompagnement en RD (6 épisodes de 140 à 223)}

L'activité professionnelle s'appuie sur les échanges avec l'enfant pour reformuler de manière plus scientifique sa question et la volonté d'identifier les « vraies questions d'enfants».

\section{L'accompagnement dans les livres (3 épisodes de 224 à 266)}

Faire trouver un ouvrage ou un chapitre et gérer le temps de recherche, voilà ce qui est en jeu dans ce moment de la RD pour les enseignants.

\section{Lire la table des matières et les articles trouvés (6 épisodes de 265 à 380)}

Ici, il s'agit pour eux de faire un état des difficultés des élèves et d'aborder la complexité des supports à utiliser tout en se projetant sur des activités possibles.

\section{Déroulement de l'exposé (6 épisodes de 381 à 496)}

Les apprentissages chez les élèves sont abordés et le lien avec d'autres moments scolaires. Cette partie du développement professionnel se termine par un retour sur les questionnements des élèves.

Ce découpage général permet d'identifier, dans un deuxième temps, comment se structurent les échanges au sein de chaque partie à partir du questionnement des chercheurs.

\subsection{Analyse du questionnement des chercheurs}

Les chercheurs n'interviennent pas beaucoup pendant l'entretien. Nous pouvons d'ailleurs observer que, parmi les 32 épisodes qui composent ce corpus, 14 sont initiés par les chercheurs, ce qui signifie que les enseignants s'approprient le dispositif et interagissent en autonomie (cf. annexe).

Plus précisément, l'entretien montre une variété d'interactions qui reposent essentiellement sur des questions mais aussi quelques constats et reformulations (Tableau 1). 
Tableau 1

Fréquence des types de questions posées par les chercheurs

\begin{tabular}{|c|c|c|c|}
\hline Chercheurs & M & $\mathrm{R}$ & C \\
\hline Question d'explicitation & 12 & 1 & 8 \\
\hline Questions en écho & 8 & 1 & 0 \\
\hline Questions de choix & 3 & 8 & 0 \\
\hline Questions de précision & 4 & 8 & 4 \\
\hline Demandes d'illustration par un exemple & 2 & 0 & 1 \\
\hline Total & 29 & 18 & 13 \\
\hline
\end{tabular}

Les questions sont les modalités les plus nombreuses. En première position viennent les demandes d'explicitation (voire reprises en écho) «qu'est-ce qui te semble important, intéressant... ? », " comment tu les fais travailler sur les livres? ». Viennent ensuite des questions sous forme de choix " est-ce que ... ou est-ce que ... ? », il s'agit alors d'obtenir pour le chercheur une réponse plutôt fermée et/ou la confirmation de ce qu'il pense comprendre. Les questions de précision prennent place aussi mais jamais directement, elles s'observent en fin d'épisode. Une dernière forme de question s'observe dans la demande d'illustration par un exemple.

Le tableau 1 montre par ailleurs que les trois chercheurs ne se saisissent pas de ce moment de la même manière. Le chercheur $\mathrm{M}$ introduit l'entretien et pose plusieurs questions ouvertes d'explicitation dont la forme majoritaire est « qu'est-ce qui se joue pour toi ? Qu'est-ce qui est important pour toi ? » Ce type de questions peut remplir une fonction de médiation qui consiste à aider le professionnel à formuler les invariants opératoires qui ont organisé son activité, invariants qui sont souvent inconscients parce qu'ils correspondent à des automatismes. Ces invariants opératoires peuvent renvoyer à l'explicitation des intentions du professionnel et la construction de sa représentation de la situation comme on le voit dans l'exemple suivant:

- 161. M : après il y a une autre question aussi c'est qu'est-ce qui te permet de dire ...d'où ça vient ... le pays le plus chaud et le plus froid et il y a une autre phase où tu expliques par rapport à la distance de la terre et le soleil ... la rotation de la terre autour d'elle-même le jour et la nuit et les saisons ... donc par rapport aux questions que tu poses, qu'est-ce qui est important pour toi en ce moment-là?

- 162. Sab : (réfléchit) Eh ... non je ne sais pas ... j'ai l'impression d'aller vraiment à la pêche et de tirer au début surtout, j'ai l'impression que Fa elle est très ... ne sait pas vraiment ce qu'elle cherche et d'essayer de lui faire redire avec ses mots ce qu'elle cherche vraiment ...eh ...

Cette fonction de médiation du chercheur pendant l'entretien d'auto-confrontation est plus visible avec les enseignants qui sont encore très proches de leur action et qui n'ont pas pris assez de recul pour réfléchir sur leurs principes d'action, comme c'est le cas de Sab.

Le chercheur $\mathrm{C}$ a une posture similaire à $\mathrm{M}$ mais les questions d'explicitation qu'il pose visent parfois à soulever un questionnement qui n'avait pas été pensé par les enseignants, ce qui peut susciter auprès de ces derniers, dans un premier temps, l'embarras et l'incompréhension. Nous présentons ici un moment où le chercheur $\mathrm{C}$ pose la question du rôle que joue la table des matières dans la démarche de la recherche documentaire. Les enseignants ne comprennent pas initialement l'intérêt de la question, mais ils se rendent comptent par la suite de la complexité de cette activité pour les élèves :

- $\quad$ 314. M : comment tu les fais travailler sur les livres?

- 315. Em : ils ont déjà leur questionnement qui sont précis et clairs pour chacun d'entre eux...ce sont des questionnements qui sont clairs, il y a un vrai questionnement sur l'islam, donc j'avais pris des encyclopédies

- 316. C: tu les avais présélectionnés? 
- 317. Em : il n'y a pas d'encyclopédies au centre de documentation, je les ai cherchés chez Sab, heu... puis.... Heu... là c'était comment on cherche une information dans un documentaire.

- 318. M : est-ce que tu avais une méthodologie en tête pour les faire chercher dans les livres ?

- 319. Em : ben d'abord chercher dans la table des matières, ou dans l'index et... heu un travail et voilà on lit le chapitre correspondant....

- 320. C : pour la petite histoire, par exemple pour piranhas, dans l'index d'un ouvrage il y avait quasiment rien et dans l'autre avec une entrée piranhas l'élève s'est retrouvé dans une grande page de texte où il fallait retrouver le mot piranhas parmi le texte; et dans le cas des volcans, le mot volcan elle l'a retrouvé partout dans l'index....la question c'est comment vous faites avec ces mots qui sont hyper pointus et du coup, c'est pas évident de les trouver et au contraire ces objets qui sont très généraux comme les volcans et qui doivent souvent revenir dans une pléthore de documents

- 321. Em : oui il y n'a pas que les index et il y a aussi les chapitres

- $322 . \quad S a b$ : elle a cherché que dans l'index?

- 323. Em: non c'est un peu long, c'est pour cela que ça prend du temps, c'est du temps nécessaire.

- 324. C : je ne dis pas que ce n'est pas nécessaire,... mais dans vos pratiques à vous qu'est-ce que vous en retenez ? Comment vous les accompagnez dans la lecture des informations?

- 325. Sab : oui effectivement sur le thème du volcan, la difficulté pour eux, en fait, moi je me suis rendu compte qu'ils ne lisent pas les en tête des chapitres, c'est-à-dire quand ils vont avoir après plusieurs pages sur les volcans, ils n'ont pas forcement de stratégies de discernement sur ... sur les sous parties, et c'est peut-être cela qu'il faut affiner justement...

Ici, la formalisation d'un savoir professionnel (repérage d'une difficulté des élèves) passe par un processus de tension et de déstabilisation, tandis que dans le cas précédent, l'explicitation d'un savoir professionnel (technique de questionnement utilisé vis-à-vis des élèves) passe par un processus de prise de conscience des invariants opératoires déjà mobilisés.

Enfin le chercheur R pose des questions quasiment exclusivement fermées aux professionnels, avec une volonté de confirmation de ses hypothèses principalement lorsqu'il s'appuie sur des éléments observés qui nécessitent des éclairages. II se situe donc moins dans une posture de médiation au sens de Vygotsky (aider l'autre à prendre conscience de son activité et à se développer professionnellement), mais plutôt dans une posture de recherche qui vise à trouver des réponses aux questions qu'il se pose. Ici des questions autour de la place des savoirs disciplinaires dans le dispositif de RD ou encore des questions autour de la place donnée au développement des compétences de l'oral au moment de l'exposé :

- 383. R: Je ne sais pas ce qui se passe par ailleurs, mais tu poses plusieurs fois ... tu dis un mot aux élèves, écrivez sur une feuille, je ne sais pas si tu t'entends le dire, ça commence très très tôt tu dis « notez les questions que vous avez pour les filles qui font l'exposé ». Et tu vas réitérer ça plusieurs fois, et personne ne pose des questions à la fin. Est-ce que c'est quelque chose qui est souvent le cas? [...]

- 394. Em : les notions sont trop compliquées à mon avis pour tout le monde... ce n'est pas des mots d'enfants... enfin voilà.

- 395. R : est-ce que dans les classes c'est souvent comme ça?

- $396 \mathrm{Em}$ : non, non d'habitude il y a des questions après les exposés

Nous sommes ici en présence de trois postures différentes qui peuvent apparaître pendant cet entretien d'auto-confrontation collectif. Les questions posées dépendent sans doute des cadres théoriques des chercheurs (analyse du travail, approches didactiques) et de leurs hypothèses concernant l'organisation du travail des enseignants et l'analyse des tâches de la RD pour les élèves. Elles révèlent que la posture du questionnement peut être plus ou moins compréhensive (ch M), formatrice (ch C) ou confirmatoire (ch R), selon le degré d'intervention dans l'analyse des moments choisis, diversité qui peut être explicitée et formalisée par avance entre les chercheurs et devenir l'objet d'une réflexion commune dans une recherche collective. 
Nous allons maintenant focaliser l'analyse sur la façon dont les professionnels s'approprient les questions posées pour échanger entre eux sur les questions cruciales soulevées par ce dispositif.

\subsection{Quelques controverses entre enseignants}

D'une façon générale, nous remarquons que les enseignants se sont saisis pleinement des échanges suscités durant ce moment collectif. Plus de la moitié des échanges correspondent à des interactions sur leurs propres pratiques. Ces échanges font apparaître des controverses concernant la gestion de l'activité de RD, controverses qui constituent l'objet même du savoir construit au sein de cette recherche collaborative. En effet, les controverses renvoient aux marges du développement du métier et sont le produit d'une réflexion commune entre les chercheurs et les enseignants. Nous en avons identifié quatre formulées sous forme de questions:

- Quelle est la place des savoirs disciplinaires dans ce dispositif qui a des objectifs d'apprentissage à la fois de contenu et de savoir-faire méthodologiques?

- Comment respecter les questions des élèves, tout en cherchant à construire des savoirs disciplinaires?

- Quel est le rôle des interactions individuelles (avec l'enseignant) et collectives (avec la classe) dans les différentes phases de ce dispositif?

- Comment guider les élèves dans la complexité des supports disposés?

Ces questions se trouvent souvent intriquées pour chaque extrait visionné. Selon les moments, il s'agit de questions dont les chercheurs sont à l'origine (principalement au début de l'entretien collectif et à chaque changement d'extraits vidéo), puis les professionnels se réapproprient ces questionnements au cours de leurs échanges selon les réponses qu'ils se font et donc les points de controverses soulevés ou les ressemblances dégagées.

On peut ainsi dire que ces controverses sont le produit d'une co-construction. La controverse concernant la complexité conceptuelle de la lecture des tables de matières pour les élèves a été provoquée par les chercheurs, par la médiation du choix des extraits vidéo soulevant des questions à ce propos. Par contre, deux controverses ont été soulevées par les enseignants seuls, à savoir les liens entre la gestion individuelle et la gestion collective des RD ainsi que la place accordée par l'enseignant au questionnement spontané de l'élève, controverses renvoyant aux débats qui animent la PF et ses différents collectifs de travail. Enfin, la controverse sur les apports et les limites du classement disciplinaire des questions spontanées des élèves correspond à une préoccupation commune des enseignants et des chercheurs, renvoyant plus profondément aux liens entre pédagogie et didactique (Reuter, 2007).

Nous allons par la suite illustrer ces controverses par deux exemples.

Premier exemple: La place des savoirs disciplinaires dans le cadre de la RD et le rôle de l'enseignant dans l'accompagnement du questionnement des élèves

Deux positions s'expriment concernant la place des savoirs disciplinaires dans le cadre de cette activité. Selon la position d'Am, le rôle de l'enseignant est d'orienter le questionnement des élèves vers une réflexion disciplinaire qui va cadrer leur recherche, économiser les moyens de réflexion utilisés et révéler les ressemblances et / ou les différences qui existent entre les thèmes de recherche proposés par les élèves:

- 90. Am : oui c'est ça mais après justement je sais que l'absence de protocole précis fait qu'on est en train de chercher et après chaque question on est déstabilisé quoi, on fait à partir de nos intuitions quoi alors que là justement je me disais sur les animaux effectivement l'idée serait de partir sur une démarche ... en tant que scientifique ou en tant que chercheur sur les animaux en général, qu'est-ce qui est intéressant à savoir ...leur alimentation etc ce qui fait que pour tous les exposés d'animaux on pourrait utiliser le premier exposé d'animal qui viendrait dans l'année quoi pour établir un protocole avec les élèves, faire quelque chose qui ne les dépasse pas, établir un protocole avec les élèves c'est-à-dire un animal, qu'est-ce qu'on a besoin de savoir sur un animal, sur l'alimentation, sur le mode de reproduction, où est-ce qu'il vit etc., ce qui fait que pour tous les autres exposés d'animaux qui reviendraient on n'aurait pas à re-réfléchir ça.

Par contre, pour Sab, trop cadrer les questions initiales des élèves signifie leur imposer un questionnement qui n'est pas le leur et qui ne va pas vraiment toucher leurs préoccupations : 
- 95. Sab : moi je trouve ça discutable en termes de recherche, je me questionne là, je me dis ... enfin ...ce qui me gêne c'est le côté normatif du coup de l'exposé ... là je parle que sur l'animal, finalement est-ce que ça vaut le coup, s'il n'a rien à battre sur combien il mesure mais pour elle c'est plus important de savoir s'il y en a un qui est rayé ou ... tu vois ...est-ce que c'est pertinent ... je ne sais pas...

- 106 - 112 Sab : alors là c'est marrant, ça me fait penser spontanément à la classification des animaux ... finalement on a bossé pendant huit séances, peut-être pas, six ou sept séances, sur la classification des animaux, ça n'a jamais ressorti dans les exposés donc voilà donc est-ce que c'est pertinent de l'imposer d'emblée tu vois?

Durant ces échanges, 2 interventions des chercheurs : I'une porte sur la mise en lien avec un exemple précédent «la carte d'identité de l'animal » (91) (aspect des savoirs disciplinaires), I'autre questionne la facilité ou non de cette proposition (aspects didactique et pédagogique) (94). Les échanges de cet extrait se déroulent alors uniquement entre les professionnels, sous l'écoute attentive des chercheurs.

Cette controverse conduit les enseignants à s'interroger sur la place à donner dans une question spontanée des enfants et sur leur rôle dans l'orientation de son questionnement. Ainsi, ils arrivent à une deuxième controverse concernant la caractérisation d'une « question d'enfant » et la façon dont elle doit être reprise par l'enseignant dans un objectif d'apprentissage (cf. annexe " émergence des questions » entre 90 et 139). Cette controverse révèle en même temps un débat développé au sein de la PF concernant le degré de respect de l'autonomie de l'élève et la gestion de la tension qui existe entre le savoir apporté par l'enseignant et le savoir construit par l'élève dans une démarche personnelle. On voit aussi apparaître les tensions qui existent entre le statut de l'enfant et celui de l'élève dans les situations d'apprentissage, tension thématisée par certains travaux de recherche (Daunay \& Fluckiger 2011).

Deuxième exemple : La place des interactions individuelles (avec l'enseignant) et collectives (avec la classe) dans les différentes phases de ce dispositif

La gestion des moments individuels, de l'interaction de face à face avec l'élève, et des moments collectifs, avec la classe, révèle également la diversité des pratiques entre les enseignants et fait apparaître une question pédagogique fondamentale, à savoir le rôle de la classe dans le développement des apprentissages, rôle thématisé et réfléchi dans le cadre de la pédagogie Freinet (Peyronie 1998).

Là aussi deux positions s'affrontent. Pour Am comme d'ailleurs pour Sab, le groupe-classe peut être utilisé pour faire avancer les questions des élèves qui présentent leur exposé ou qui cherchent leur sujet d'exposé. Il peut aussi être utilisé pour réduire I'autorité de l'enseignant en tant que porteur de savoir et donner du pouvoir au collectif des élèves.

Pour Em en revanche, gérer les questions des élèves en grand groupe représente un danger parce qu'elle a peur de ne pas pouvoir maîtriser la diversité des interventions des élèves. Elle préfère donc passer par une phase de questionnement individuel, de face à face, avec l'élève pour l'aider à stabiliser sa question, avant de passer dans une phase de travail collectif pendant le moment de l'exposé. On remarque que le chercheur ici ne fait que poser des questions d'explicitation pour mieux comprendre ce qu'Em veut dire :

- 53. Em : moi je ne fais plus cette démarche collective, je fais comme pour la recherche maths, donc si la question est pourquoi les tulipes c'est beau ban on va prendre cinq minutes, on va chercher ensemble...

- $\quad$ 54. $\quad R$ : ensemble tu veux dire...

- 55. Em : tous les deux voilà c'est essayer de comprendre, moi j'essaye de comprendre ... on construit ensemble un questionnement...

- 56. M: pour comprendre quoi exactement?

- 57. Em : pour comprendre il y a quoi derrière ce questionnement, il y a quoi derrière la tête de l'enfant

- 58. M: dans la tête de l'enfant?

- 59. Em : ... voilà pourquoi tu t'es posé cette question, qu'est-ce que tu trouves beau, c'est vraiment une discussion et puis je prends des notes en même temps et on affine les questions... c'est trop compliqué pour moi, c'est trop de questions de pourquoi voilà c'est trop de questions scientifiques et moi je n'arrive pas à le gérer c'est trop de choses et ... par contre à la fin de l'exposé ils peuvent poser d'autres questions, ils peuvent élargir. Mais moi je n'arrive pas à gérer l'abondance des questions, très larges comme ça et puis ça peut sortir, on peut sortir du questionnement 
initial de l'enfant, I'histoire de la tulipe qui est belle beh ça peut être important pour ce moment-là et puis après on passe sur autre chose sur les plantes en général et ça sera le questionnement qui va être présenté à l'exposé, voilà.

L'analyse montre que les controverses sont le produit d'une co-construction. Si le choix des extraits vidéo et surtout les visées de ces derniers a un impact sur les formes d'échanges avec les professionnels, ces derniers s'approprient de façon spécifique les questions posées selon leurs propres préoccupations. Les pratiques d'explicitation sont fécondes pour les accompagner dans la formulation de leurs automatismes professionnels. Avec ici un passage par la description, puis par les échanges chercheurs/ enseignants, une visée plus constructive des pratiques personnelles et collaboratives apparaît progressivement. Les vidéos relatives à des prises de conscience de difficultés non perçues supposent de la part des chercheurs plus d'illustrations, d'exemples précis pour conduire les enseignants à se saisir de ce moment. II s'agit alors d'une volonté de formation du chercheur pour faire naître le besoin de travailler sur un nouveau savoir professionnel. Il est parfois très difficile pour certains enseignants de pouvoir penser ces éléments de leurs pratiques et de les faire sortir de l'évidence, donc de déstabiliser leurs représentations.

\section{Conclusion}

Cette recherche collaborative a effectivement développé les trois points proposés par Desgagné (1997), à savoir une coconstruction de l'objet d'enseignement « recherche documentaire », un retour sur l'analyse de ces moments de RD et des possibilités d'adaptations futures, un temps de formation pour les praticiens principalement sur la lisibilité des textes documentaires. C'est également une vraie médiation enseignants/ chercheurs dans la construction réciproque des questionnements professionnels concernant le RD. Bien que les échanges soient initiés par les chercheurs à partir des extraits de vidéos, ce collectif d'enseignants qui a l'habitude de travailler ensemble se nourrit aussi bien des échanges des chercheurs/praticiens que des échanges praticiens/praticiens. La réflexion sur l'activité réelle débouche souvent sur la projection dans une activité possible et des pistes d'évolution et d'adaptation des pratiques. Nous observons d'ailleurs une volonté de poursuivre ce travail avec l'aide des chercheurs qui se dégage à la fin de l'année d'observation.

Notre analyse montre l'intérêt d'un regard discursif de l'entretien d'auto-confrontation qui met en évidence les faits de langue contribuant à la fois à la construction des objets du discours et des rôles et places discursives : utilisation des fonctions du langage, diversité des postures et des questionnements. L'analyse de ces faits de langue présente plusieurs intérêts. Premièrement, certaines pratiques professionnelles sont difficiles à mettre en mots, "ce truc » comme dirait Am relève d'une prise en compte d'une connaissance quotidienne au sens de Vygotsky. Nous pouvons dire que cette difficulté à nommer précisément l'activité recoupe les théorèmes en actes de Vergnaud et témoigne de l'influence de ce temps d'entretien d'auto confrontation sur les savoirs professionnels et leur développement. Deuxièmement, les faits de langue montrent que ce moment constitue en soi une autre activité d'interaction sociale qui se superpose à la première (celle qui est filmée) en créant des « positions de savoir » qui se négocient constamment aussi bien avec le chercheur (Morrissette et Desgagné 2009) qu'entre les enseignants. L'analyse discursive semble centrale pour montrer l'enjeu méthodologique de la gestion de cette tension par le chercheur qui veille à ce que l'activité de référence et son organisation reste la préoccupation centrale du groupe pendant le déroulement de l'entretien. Enfin, l'analyse des modalités de questionnements des chercheurs montre que les postures peuvent varier d'un chercheur à l'autre, variété qui mériterait d'être explicitée et thématisée pour caractériser davantage l'entretien d'autoconfrontation collectif, ses biais, ses limites et les vigilances à mettre en place dans le cadre d'une recherche collaborative.

\section{Références bibliographiques}

Boyer, C., Pagoni, M. (2015). Interroger la variabilité des pratiques de trois enseignants dans le cadre de la recherche documentaire à l'école primaire. Communication présentée dans le colloque international Condition(s) enseignante(s), Conditions pour enseigner, Université Lyon 2, France, du 8- 10 janvier.

Catroux, M. (2002). Introduction à la recherche-action : modalités d'une démarche théorique centrée sur la pratique. Recherche et pratiques pédagogiques en langues de spécialité, XXI (3). Téléaccessible à : : http://apliut.revues.org/4276

Clot, Y. (1999). La fonction psychologique du travail. Paris : PUF.

Clot, Y., Faïta, D., Fernandez, G. \& Scheller, L. (2001). Entretiens en auto-confrontation croisée : une méthode en clinique de I'activité. Education Permanente, 146(1), 17-25.

Daunay, B., Fluckiger C. dir. (2011). Enfant-élève-apprenant. Villeneuve d'Ascq : Presses universitaires du Septentrion diffusion. 
Desgagné, S. (1997). Le concept de recherche collaborative : I'idée d'un rapprochement entre chercheurs universitaires et praticiens enseignants. Revue des sciences de l'éducation, 23(2), 371-393.

Faïta, D. Vieira, M. (2003). Réflexions méthodologiques sur l'auto-confrontation croisée. DELTA,19(1) Téléaccessible à : http:// www.scielo.br/scielo.php?script=sci_arttext\&pid=S0102-44502003000100005

Kahn, S., Hersant, M. et Orange-Ravachol, D. (2010). Savoirs et collaborations entre enseignants et chercheurs en éducation. Recherches en éducation, hors série(1), 147 p. [En ligne] http://www.recherches-en-education.net/IMG/pdf/REE-HS-no1. pdf

Pagoni, M. (2014). Construire la signification des situations en conseil de coopérative. Quel questionnement de l'enseignant? In Pagoni M. coord., Ecole(s) et culture(s): perspectives internationales. Savoirs scolaires, pratiques sociales et significations (p. 259-278), Bruxelles: Petre Lang, collection Transversales.

Pastré, P. (2011). La didactique professionnelle. Approche anthropologique du développement chez les adultes. Paris : PUF.

Peyronie, H. dir. (1998). Freinet, 70 après. Une pédagogie du travail et de la dédicace ? Caen : Presses Universitaires de Caen.

Morrissette, J., \& Desgagné, S. (2009). Le jeu des positions de savoir en recherche collaborative : une analyse. Recherches qualitatives, 28(2), 118-144.

Morrissette, J. (2013). Recherche-action et recherche collaborative : quel rapport aux savoirs et à la production de savoirs ? Nouvelles pratiques sociales, 25(2), 35-49. Téléaccessible à : http://www.erudit.org/revue/nps/2013/v25/n2/index.html

Reuter, Y. dir. (2007). Une école Freinet; fonctionnement et effets d'une pédagogie alternative en milieu populaire. Paris : L'Harmattan.

Schön, D.A. (1983). Le praticien réflexif. À la recherche du savoir caché dans l'agir professionnel (J. Heynemand \& D. Gagnon, trad.). Montréal : Logiques.

Schneuwly, B., Dolz J., Ronveaux, Ch. (2006). Le synopsis : un outil pour analyser les objets enseignés. Dans G. Perrin et Y. Reuter (dir.). Les méthodes de recherche en didactiques, (p.75-189), Villeneuve d'Ascq : Septentrion.

Vergnaud, G. (1990). La théorie des champs conceptuels. Recherches en Didactique des Mathématiques, 10(2.3), 133-170.

Vinatier, I. (2009). Pour une didactique professionnelle de l'enseignement. Rennes: Presses Universitaires de Rennes.

Vygotsky, L. (1985). Pensée et langage (Traduit du Russe par Françoise Sève). Paris : La dispute. 


\section{Annexe : Découpage de l'entretien d'auto-confrontation collectif en épisodes}

\begin{tabular}{l|l|}
$\begin{array}{c}\text { Tours de } \\
\text { parole }\end{array}$ & \multicolumn{1}{|c}{ Titre de l'épisode } \\
\hline $1-3$ & Présentation de la consigne du travail \\
\hline $4-21$ & $\begin{array}{l}\text { Premier extrait : l'émergence de questionne- } \\
\text { ment dans la classe d'Am Contextualisation }\end{array}$ \\
\hline $22-36$ & $\begin{array}{l}\text { Comprendre et gérer le questionnement de } \\
\text { I'enfant, selon Am. }\end{array}$ \\
\hline $37-51$ & $\begin{array}{l}\text { Nécessité de construire un protocole de tra- } \\
\text { vail en commun pour classer les questions des } \\
\text { élèves dans un questionnement disciplinaire, } \\
\text { selon Am }\end{array}$ \\
\hline $52-63$ & $\begin{array}{l}\text { Identifier le sens des questions des élèves avec } \\
\text { une organisation individuelle ou collective (avec } \\
\text { toute la classe). }\end{array}$
\end{tabular}

64 - 81 Quel intérêt et quel moment de classement des questions?

82 - $89 \quad$ Prendre en compte la connaissance de l'enfant qui pose la question, selon Am.

90 - 105 Retour sur la nécessité de construire un protocole et échange de pratiques

106 -134 Retour sur le sens de la question de l'élève et sa gestion individuelle ou collective.

135-139 Retour sur l'expérience de cette année, lien entre contenu des savoirs et méthodologie de RD

\begin{tabular}{l|l} 
& RD \\
\hline $140-160$ & $\begin{array}{l}\text { Deuxième extrait : L'accompagnement de Sab } \\
\text { pendant la RD Contextualisation }\end{array}$ \\
\hline $161-170$ & $\begin{array}{l}\text { La spontanéité de l'interaction avec l'élève se- } \\
\text { lon Sab }\end{array}$ \\
\hline $171-176$ & Contextualisation
\end{tabular}

Personne à l'origine de l'épisode

Chercheur M: «Qu'est-ce qui se joue pour vous dans ces extraits, est-ce que vous avez un objectif...»

Chercheur R qui présente l'extrait

Chercheur M: «Si tu veux commenter, qu'est-ce qui se joue pour toi en fait dans cette phase de questionnement, qu'estce qui est important ... »

Chercheur C: "Et les autres collègues, vous avez des commentaires par rapport à ce qui est présenté... ou des questions à poser ....

Directrice: «Donc une question comme cela pourquoi la tulipe c'est beau on ne peut pas vraiment la classifier en termes scientifiques ...»

Sab: "Moi par rapport à ce que tu disais Am, je n'ai pas entendu la même chose ... parce que le classement par thématique j'ai l'impression que tu parlais plutôt de types de questions ....

Am: « Mais à mon avis ce qui manque, ce qui est déterminant dans mon jugement c'est la connaissance de l'élève, quand je dis à M. le côté sombre et éclairé, je connais l'élève et je sais qu'il est capable d'un questionnement très pointu comme ça ...».

Am: « Oui c'est ça mais après justement je sais que l'absence de protocole précis fait qu'on est en train de chercher et après chaque question on est déstabilisé quoi ...»

Sab: « Alors là c'est marrant, ça me fait penser spontanément à la classification des animaux...»

Sab: «Moi ce qui me vient à l'esprit c'est que quelque part $c^{\prime}$ 'est notre première année où c'est mieux organisé par rapport à l'année dernière, moi j'ai l'impression que ça a mieux marché que l'année dernière ... ».

Ch M: «On passe à un autre extrait?»

Ch M: «par rapport aux questions que tu poses, qu'est-ce qui est important pour toi en ce moment-là? »

Ch C: «Ce jour-là le centre de documentation était ou devait être en travaux et vous étiez dans la salle informatique » 


\begin{tabular}{|c|c|c|}
\hline $177-184$ & Internet et accompagnement individuel & $\begin{array}{l}\text { Em: " et finalement je trouve cela intéressant d'habitude on } \\
\text { ne va jamais sur internet directement mais là tu y vas dès le } \\
\text { début et en fait vous cherchez ensemble et vous heurtez aux } \\
\text { écueils d'internet ensemble... " }\end{array}$ \\
\hline $185-207$ & $\begin{array}{l}\text { Transformer la question de l'élève en question } \\
\text { conceptuelle - généralisation }\end{array}$ & $\begin{array}{l}\text { Em: «Donc on a un questionnement à elle, presque intime et } \\
\text { on arrive à une notion plus large... » }\end{array}$ \\
\hline $208-223$ & Les « vraies questions » des élèves & $\begin{array}{l}\text { Sab: « ..la question quel est le pays le plus chaud quel est le } \\
\text { pays le plus froid c'est une question qui a émergé du débat } \\
\text { collectif dans ta classe? ... parce que ça c'est une vraie ques- } \\
\text { tion...» }\end{array}$ \\
\hline $224-233$ & $\begin{array}{l}\text { Phase de transition : Accompagnement de la } \\
\text { recherche dans un livre }\end{array}$ & $\begin{array}{l}\text { Ch C: «Ce qui était intéressant dans vos échanges c'est ce } \\
\text { que peut apporter le livre... c'est-à-dire que si vous n'allez pas } \\
\text { directement sur Internet... Comment justement ce passage } \\
\text { de chaud-froid, de température donc le point de départ en } \\
\text { fait....Qu'est-ce qui se serait passé ? Comment l'accompa- } \\
\text { gnement aurait pu se passer pour trouver la réponse sur un } \\
\text { ouvrage?» }\end{array}$ \\
\hline $234-243$ & Difficulté à trouver les documents & $\begin{array}{l}\text { Sab: «Par contre il y a des questions c'est vrai comme le } \\
\text { ballon d'or... pas de documents sur le ballon d'or en BCD } \\
\text { c'est clair » }\end{array}$ \\
\hline 244 à 266 & Nécessité de partager les pratiques de classe & $\begin{array}{l}\text { Am: " parce que le problème en plus ce jour là je me sou- } \\
\text { viens, pendant que moi je discutais les autres discutaient se } \\
\text { tournaient les pouces ils attendaient, c'était fini... il fallait cla- } \\
\text { rifier au niveau des questions » }\end{array}$ \\
\hline $267-325$ & $\begin{array}{l}\text { Troisième extrait: La recherche dans un livre et } \\
\text { la table de matières } \\
\text { Contextualisation et introduction du question- } \\
\text { nement }\end{array}$ & $\begin{array}{l}\text { Ch C: « II y a plein de choses en fait. vous avez fait émer- } \\
\text { ger tous les moments qui peuvent vous poser soucis, moi ce } \\
\text { que j'ai retenu du dernier travail ...c'est comment les élèves } \\
\text { s'approprient..., comment tu les emmenais à regarder les } \\
\text { index...» }\end{array}$ \\
\hline $326-333$ & $\begin{array}{l}\text { Difficultés des stratégies de lecture des élèves } \\
\text { dans le texte }\end{array}$ & $\begin{array}{l}\text { Ch C: "qu'est-ce que vous en retenez ? comment vous les } \\
\text { accompagnez comme on a vu tout à l'heure avec internet... } \\
\text { dans la lecture des informations? " }\end{array}$ \\
\hline $334-341$ & $\begin{array}{l}\text { Adapter les supports à la lecture, étayer le choix } \\
\text { des élèves }\end{array}$ & $\begin{array}{l}\text { Am: « moi je lisais sur le site de classe Freinet qui font ça } \\
\text { depuis longtemps, ils bloquaient les documents, ils intro- } \\
\text { duisaient progressivement d'autres documents.... c'était au } \\
\text { départ sur ce document parce qu'on apprend à maitriser sa } \\
\text { table des matières }\end{array}$ \\
\hline $342-351$ & $\begin{array}{l}\text { Choix des supports a priori et } \\
\text { questionnement des élèves }\end{array}$ & $\begin{array}{l}\text { Em: "ça implique de sélectionner les doc en amont, donc } \\
\text { forcément toutes les questions des enfants..... " }\end{array}$ \\
\hline $352-362$ & $\begin{array}{l}\text { De l'organisation des procédures à un question- } \\
\text { nement sur la démarche didactique }\end{array}$ & Directrice: « question d'organisation ...» \\
\hline $363-380$ & $\begin{array}{l}\text { Problème de l'organisation du dispositif et du- } \\
\text { rée }\end{array}$ & $\begin{array}{l}\text { Ch } \mathrm{R} \text { : « mais ce que tu sembles dire, c'est qu'il y a perte de } \\
\text { temps ...» }\end{array}$ \\
\hline
\end{tabular}




\begin{tabular}{|c|c|c|}
\hline $381-403$ & $\begin{array}{l}\text { Troisième extrait : I'exposé dans la classe d'Em } \\
\text { Présentation du film } \\
\text { Contextualisation et introduction } \\
\text { du questionnement par Em }\end{array}$ & $\begin{array}{l}\text { Em: « là c'était mon problème quand je n'avais pas en charge } \\
\text { les exposés ...je n'ai pas pu vérifier qu'elles connaissent les } \\
\text { mots, qu'elles connaissent ce dont elles parlent. Donc ça me } \\
\text { posait problèmes quand les exposés étaient uniquement pris } \\
\text { en charge par d'autres collègues c'est dur de maîtriser tout } \\
\text { ce qui va être dit » }\end{array}$ \\
\hline $404-411$ & Formulation des questions après l'exposé & $\begin{array}{l}\text { Ch R: «Mais là au niveau de ce qui se passe à l'oral, Est-ce } \\
\text { qu'il y a un enjeu au niveau de la performance même de l'oral } \\
\text { ? Qu'est-ce qui est important là ce moment-là? » }\end{array}$ \\
\hline $412-424$ & Les critères d'un exposé réussi & $\begin{array}{l}\text { Sab: «c'est vrai que les questions des enfants permettent de } \\
\text { voir ce que l'élève qui a fait la recherche a vraiment compris } \\
\text { surtout si il est capable d'y répondre. " }\end{array}$ \\
\hline $425-444$ & Les exposés et les autres moments scolaires & $\begin{array}{l}\text { Ch } \mathrm{M}: \text { «....il y a d'autres moments où vous faites des sciences, } \\
\text { de l'histoire....? }\end{array}$ \\
\hline $445-469$ & $\begin{array}{l}\text { L'émergence des exposés - la notion de «for- } \\
\text { mule intermédiaire » }\end{array}$ & $\begin{array}{l}\text { Am: « .....il y a toujours au départ des parts de contraintes et } \\
\text { des parts de libertés, on va progressivement effacer la part } \\
\text { de contraintes mais ça doit être à mon avis.... » }\end{array}$ \\
\hline $470-496$ & $\begin{array}{l}\text { Partager les savoirs méthodologiques pour les } \\
\text { élèves }\end{array}$ & $\begin{array}{l}\text { Ch R: «Une dernière question Em, quand tu poses la ques- } \\
\text { tion de la méthode est ce que c'est une question pour toi ou } \\
\text { est-ce que c'est une question pour la classe? }\end{array}$ \\
\hline 497- 510 & $\begin{array}{l}\text { Conclusion et perspectives: Echange sur l'aide } \\
\text { des chercheurs pour l'an prochain }\end{array}$ & Sab: « On continue l'année prochaine?» \\
\hline
\end{tabular}

